Memorias del VII Encuentro Nacional de Experiencias en la Enseñanza de la Biología y la Educación Ambiental y II Congreso Nacional de Investigación en la Enseñanza de la Biología

\title{
LA REPRESENTACIÓN DEL CONCEPTO ALIMENTACIÓN A PARTIR DE LOS DESCRIPTORES DEL ENTORNO NATURAL.
}

\section{THE FEEDING CONCEPT REPRESENTATION STARTING FROM THE DESCRIPTORS OF THE NATURAL ENVIRONMENT.}

\section{Diana Marcela Bautista Sánchez ${ }^{1}$}

Andrés Arturo Venegas Segura²

\section{Resumen}

El presente artículo expone parte de los resultados obtenidos en el trabajo de investigación titulado, "Representaciones del concepto alimentación de niños y niñas Citadinos, Rurales y Wayúu en la clase de ciencias naturales: una mirada cultural". Este escrito hace referencia a los múltiples elementos del mundo natural que los estudiantes de estas procedencias vinculan en sus representaciones del concepto alimentación. Dichos elementos o descriptores permiten inferir que el Hombre establece relaciones con el mundo natural partiendo de su cultura y contexto a fin de conocerlo y comprenderlo para llegar a aprovecharlo y sobrevivir en y por él. De ahí la importancia de su reconocimiento como punto de partida para entablar dinámicas de enseñanza y aprendizaje en el marco de la multiculturalidad, donde la clase de ciencias se constituye como una herramienta para la generación e impulso de procesos de empoderamiento cultural y decolonialización del conocimiento. De esta manera, lo aprendido contribuye efectivamente en la solución de problemas partiendo del contexto cultural y de la interpretación del mundo que ostentan los niños y niñas de las diversas comunidades asistentes a la escuela.

Palabras clave: Citadino, Rural, Wayúu, enseñanza de las ciencias, multiculturalidad, empoderamiento, representación, alimentación.

\section{Abstract}

This paper presents part of the results of the research work entitled, "Representations of the concept feeding of childrens Urban, Rural and Wayuu in science class: A cultural look". This paper refers to the multiple elements of the natural world those students from these backgrounds linking into their representations of feeding concept. These elements or descriptors to infer that

\footnotetext{
${ }^{1}$ Licenciada en Biología, Universidad Distrital Francisco José de Caldas. Centro de investigaciones y desarrollo científico.

${ }^{2}$ Dr. Doctorado Interinstitucional en Educación. Centro de investigación y desarrollo científico.
} 
Memorias del VII Encuentro Nacional de Experiencias en la Enseñanza de la Biología y la Educación Ambiental y II Congreso Nacional de Investigación en la Enseñanza de la Biología

Man builds relationships with the natural world starting from their culture and context in order to know and understand to get to use it and survive in and through him. Hence the importance of its recognition as a starting point to establish dynamics to teaching and learning in the framework of multiculturalism, where science class is constituted as a tool for the generation and promotion of cultural empowerment processes and decolonization of knowledge. Thus, it learning contributes effectively in solving problems based on the cultural context and interpretation of the world of children's diverse communities attending the school. Keywords: Urban, Rural, Wayuu, teaching of science, multiculturalism, empowerment, representation, feeding.

\section{Introducción}

Múltiples investigadores han identificado como inadecuada la educación en ciencias para grupos minoritarios debido a su carácter descontextualizado. Así, autores como Hewitt (1905) explican que los colegios coloniales en Estados Unidos atribuyen una inferioridad genética a las comunidades de nativos americanos e inmigrantes como manera de justificar su fracaso a nivel escolar.

En este sentido, Philips (1976) se refiere a la deficiencia académica como un resultado sujeto a la diferencia culturalmente significativa existente en los ambientes de aprendizaje en que se desenvuelven los estudiantes, pues no hay una correlación entre la escuela y otros contextos como el hogar, la relación entre pares, etc.

Este aspecto presupone que el currículo en ciencias debe acercarse a las formas propias de aprender de los estudiantes, a fin de reducir los conflictos culturales generados entre las comunidades y la escuela occidental.

Ogbu (1982) plantea que el éxito escolar de los estudiantes se relaciona con su cultura, en este sentido, señala como los estudiantes pertenecientes a la clase media en Estados Unidos tienen un mayor éxito escolar debido a que la cultura que se exhibe en la enseñanza es muy similar a su cultura de origen, caso contrario con respecto a los niños que provienen de minorías, los cuales no alcanzan tal éxito pues provienen de otras culturas, es decir, la brecha cultural existente entre lo enseñado en la escuela y sus culturas, genera un proceso de discontinuidad cultural que inminentemente conlleva al fracaso académico de los estudiantes. Este panorama conduce a considerar que el reconocimiento de los saberes culturales de las comunidades en la escuela y la clase de ciencias, implica la incorporación de las dimensiones culturales de los estudiantes en los planes de estudios, aspecto presente en la investigación de Riggs (2005) con algunos grupos indígenas de los Estados Unidos en la enseñanza de la geología, donde el autor llama la atención sobre las ventajas de enfocar el trabajo de aula con respecto a los antecedentes culturales de los grupos indígenas. 
Memorias del VII Encuentro Nacional de Experiencias en la Enseñanza de la Biología y la Educación Ambiental y II Congreso Nacional de Investigación en la Enseñanza de la Biología

El caso colombiano no dista mucho del panorama antes expuesto, pues aunque la constitución política de 1991 proclama y ratifica la presencia de grupos minoritarios sobre el territorio nacional (indígenas, campesinos, raizales, afrocolombianos, rom, entre otros), la educación en ciencias sigue impartiéndose fundamentada en los preceptos de occidente, recalcando los valores del cientificismo e invisibilizando los saberes y conocimientos ancestrales y tradicionales propios de estas culturas.

En este sentido, el trabajo de investigación, "Representaciones del concepto alimentación de niños y niñas Citadinos, Rurales y Wayúu en la clase de ciencias naturales: una mirada cultural" pretendió el reconocimiento de saberes y conocimientos en torno al concepto alimentación teniendo en cuenta las diferencias culturales manifestadas por los estudiantes a través de sus representaciones. Éste se enmarcó en un enfoque transcultural (Cross cultural) al considerar importante examinar y reflexionar ante las consecuencias de los objetivos educativos diseñados y dirigidos a colectividades donde existen grupos culturalmente diferenciados, a fin de plantear las diferencias culturales y contextuales como dimensión fundamental para promover el empoderamiento cultural y la deconolización del conocimiento a través de la clase de ciencias.

En consecuencia, los resultados expuestos en el presente artículo además de exponer las representaciones en torno al concepto alimentación a partir de los descriptores presentes en el entorno natural, revelan la diversidad y semejanza culturales existentes en torno a temáticas tratadas en la clase de ciencias naturales, cuya vinculación en el aula es el paso a seguir para desarrollar una educación incluyente, enmarcada en los principios de igualdad y diferencia, significativa para los estudiantes y generadora de interacción cultural positiva entre los diferentes.

\section{Metodología}

La investigación fue desarrollada dentro del Santuario de Fauna y Flora Los Flamencos, en el Corregimiento de Camarones, Dpto. de La Guajira, Colombia. Éste se ubica $17 \mathrm{~km}$ al sur de la ciudad de Riohacha y se aleja $2 \mathrm{~km}$ de la costa del mar Caribe donde se encuentran las llamadas bocas de Camarones. Su población está constituida por la mezcla entre indígenas Wayúu, Afro colombianos y habitantes procedentes de diversas regiones de la costa Caribe. Allí se encuentra la Institución Educativa Nacional Luis Antonio Robles, la cual atiende tanto educación básica primaria, como básica secundaria y media vocacional, destacando un énfasis en ecoturismo (Parques Nacionales Naturales, de Colombia, 2007; Bautista: diario de campo). 
Memorias del VII Encuentro Nacional de Experiencias en la Enseñanza de la Biología y la Educación Ambiental y II Congreso Nacional de Investigación en la

\section{Enseñanza de la Biología}

El diseño metodológico planteado para realizar la investigación contempló tres fases a saber: A) Fase de relevamiento bibliográfico: incluyó una fundamentación teórica cuyos fines fueron: 1) definir el fenómeno a estudiar, establecer un marco conceptual general para el estudio, determinar los antecedentes en este tipo de investigación; y 2) fundamentar el diseño del instrumento de compilación de información a partir de la triangulación de expertos. B) Fase de campo: incluyó el proceso de concertación a distancia con la comunidad a fin de agendar la visita; reconocimiento de la comunidad de estudio; aplicación del instrumento de compilación de información, construcción del diario de campo a partir de la observación participante. C) Fase de análisis e interpretación: incluyó la caracterización inicial de la información obtenida; elaboración de una matriz global; definición de las categorías de análisis y contrastación a partir de la información obtenida, análisis de las ilustraciones y descripciones en el marco de las categorías encontradas a fin de vislumbrar las representaciones.

Con respecto al instrumento aplicado, cabe resaltar que se dividió en dos partes pues incluyó una ficha para adelantar la caracterización socio-demográfica de cada estudiante, a fin de compilar información referente al género, la edad, el curso y la procedencia cultural. Una segunda parte, incluyó un enunciado y el espacio para desarrollar el ejercicio solicitado. Es importante aclarar que el encabezado del instrumento fue diseñado de manera que el estudiante pudiese asumir el rol de profesor, ello con el fin de motivar al niño o niña a otorgar explicaciones (gráficas y escritas) amplias, sin temor a ser juzgado. Asimismo, el encabezado buscó mostrar la importancia que el investigador adjudicó a las respuestas, al adquirir un papel de aprendiz con respecto a los estudiantes. De esta manera, se pretendió que los niños y niñas comprendieran que su voz sería la protagonista.

Es fundamental aclarar aquí que la representación fue abordada desde la perspectiva de Hall (1997) quien plantea las prácticas de representación como un proceso clave del "circuito cultural", por lo cual su vinculación dentro de los estudios culturales resulta notable al configurar una mediación que hace posible la inteligibilidad del mundo. De esta manera, la representación según Hall (1997) conecta el sentido al lenguaje y a la cultura, "...Representación significa usar el lenguaje para decir algo con sentido sobre, o para describir de manera significativa el mundo a otras personas..." (p. 2). En consecuencia, la representación implica el uso de signos, ya sean palabras escritas, sonidos dichos, o imágenes visuales. De ahí que el instrumento de compilación de información, contemplara por un lado, la realización de un dibujo, y por el otro, la descripción escrita del mismo.

Ahora bien, es de suma importancia tener en cuenta que representar implica formar conceptos de cosas percibidas, personas, objetos materiales, animales, 
Memorias del VII Encuentro Nacional de Experiencias en la Enseñanza de la Biología y la Educación Ambiental y II Congreso Nacional de Investigación en la

\section{Enseñanza de La Biología}

alimentos, etc. Sin embargo, también es posible formar conceptos de cosas abstractas, no tangibles, como el amor. De ahí que, sea posible pensar que una representación puede referirse a aquello que se ha observado como también denotar algo que nunca se ha visto o que fue inventado, lo cual en términos educativos denota el reconocimiento de características fundamentales del universo discursivo de los estudiantes. Así pues, desde esta perspectiva los signos representan los conceptos y las relaciones conceptuales entre ellos, lo cual constituye aquello que se denomina sistemas de sentido de una cultura, aspecto que entra en consonancia con la definición de cultura establecida por Geertz (1987).

Respecto a la fase de análisis e interpretación es válido mencionar que ésta se fundamentó en los postulados de Ricoeur $(2000,2006)$ y Eco $(1953,1986)$ pues por una parte, la teoría de la interpretación planteada por Ricoeur $(2000 ; 2006)$ se orienta hacia el texto, el cual es considerado como un lenguaje escrito, lo cual hace posible una aproximación interpretativa más acertada frente al significado que los estudiantes atribuyen a las representaciones del concepto alimentación por medio de las descripciones (lenguaje escrito, oraciones) que acompañan sus ilustraciones, reconociendo además las referencias que las fundamentan.

Por otro lado, los planteamientos de Eco $(1953,1986)$ sugieren que todo acto de comunicación funciona con la emisión de mensajes basados en códigos subyacentes. Dichos códigos se definen como sistemas de símbolos que permiten representar y transmitir una información deseada de un emisor a un receptor o "de una fuente a un punto de destino" (Eco, 1986, p. 9). Este aspecto entró en consonancia con el trabajo, pues a través de la imagen (entendida desde esta perspectiva como un conjunto articulado de símbolos) los estudiantes manifestaron sus representaciones del concepto alimentación.

Asimismo, Eco (1986) vincula la cultura dentro de las representaciones, pues siguiere que al momento en que un emisor se dirige a alguien a través de una palabra, un gesto, un signo, o un código (conjunto de símbolos) hace uso de una serie de reglas establecidas para hacerse comprensible ante el otro. Dicho conjunto de reglas existe en todo proceso de comunicación y se establece a partir de una convención cultural. En este sentido, el reconocimiento de las representaciones del concepto alimentación pone en evidencia presupuestos de orden cultural que los estudiantes manifiestan a la hora de comunicar dicha representación.

Ahora bien, el análisis y la interpretación incluyeron una caracterización inicial de la información obtenida a través del instrumento. Para ello se asignó un código a cada uno de los estudiantes. Los instrumentos fueron digitalizados y organizados en fichas las cuales contemplaron el código asignado, la procedencia del 
Memorias del VII Encuentro Nacional de Experiencias en la Enseñanza de la Biología y la Educación Ambiental y II Congreso Nacional de Investigación en la Enseñanza de la Biología

estudiante, la ilustración realizada junto a la transcripción literal de las descripciones escritas (si existieron), y un análisis inicial. El objetivo de este ejercicio fue identificar aquello que fue representado y su relación con el contexto, las experiencias, los saberes tradicionales y la formación científico-escolar de los estudiantes. En el caso de los dibujos, se vislumbraron los elementos presentes en términos de contenido (códigos icónicos, signos icónicos y códigos de reconocimiento). Hecho similar ocurrió con las descripciones que acompañan las ilustraciones, pues su transcripción literal permitió reconocer características del universo discursivo de los estudiantes en torno al concepto alimentación, poniendo en evidencia el significado que ostentan las representaciones.

Tras el proceso de análisis inicial, se establecieron las categorías de análisis con las cuales se realizó la interpretación posterior. Éstas se configuraron a la vez como pautas de contrastación que permitieron establecer similitudes y diferencias en las representaciones de los estudiantes según su procedencia: Indígenas Wayúu, Rurales y Citadinos. De esta manera emergieron las categorías de análisis dentro de los cuales se encuentra aquella denominada "Descriptores del entorno natural". Por último, se procedió a tabular y organizar los elementos identificados (tanto en las ilustraciones como en las descripciones) dentro de dichas categorías, ejercicio que permitió conformar una matriz global que contempló una ficha estadística y una ficha descriptiva. A partir de la información estadística se identificó la presencia o ausencia de cada una de las categorías de análisis dentro de las ilustraciones y descripciones realizadas por cada niño o niña (1: presente; 0: ausente). Así, fue posible establecer la cantidad de estudiantes según su procedencia, que manifestaron cada categoría en términos de número y porcentaje. Es valioso aclarar, que la matriz global fue dividida de esta manera con el fin de favorecer el rigor metodológico de la investigación, al exponer datos cuantitativos que apoyan la constitución cualitativa del trabajo.

\section{Resultados y discusión}

A partir de la ficha de caracterización socio-demográfica del instrumento fue posible determinar las características cuantitativas del grupo de estudio. Éste, comprendió el grado 501 de la Institución Educativa Luis A. Robles, conformado por 26 estudiantes (16 niños, 10 niñas) con edades entre los 11 y los 16 años. Teniendo en cuenta la procedencia de los estudiantes se identificaron 3 grupos diferenciados: indígenas Wayúu, Rurales y Citadinos. Los estudiantes de procedencia indígena Wayúu presentaron el mayor número de miembros (17 estudiantes) seguidos por el grupo de estudiantes de procedencia Rural (5 estudiantes) y el grupo de procedencia Citadina (4 estudiantes).

La categoría "descriptores del entorno natural” emergió a partir del análisis de la información obtenida. Ésta hace referencia a los múltiples elementos del mundo 
Memorias del VII Encuentro Nacional de Experiencias en la Enseñanza de la Biología y la Educación Ambiental y II Congreso Nacional de Investigación en la

\section{Enseñanza de la Biología}

natural que los estudiantes vinculan en sus representaciones del concepto alimentación. Dichos elementos permiten inferir que el Hombre establece relaciones con el mundo natural a fin de conocerlo y comprenderlo para llegar a aprovecharlo y sobrevivir en y por él. Estos elementos fueron definidos a partir de los dibujos y afirmaciones de los estudiantes participantes y evidencian perspectivas culturales diversas que resaltan la presencia, importancia y significado del entorno natural en la representación del concepto alimentación de los niños y las niñas.

Así, fue posible determinar que el $42 \%$ de los estudiantes relacionaron en sus dibujos y afirmaciones elementos descriptores del entorno natural. Este porcentaje correspondió a seis (6) estudiantes de procedencia Indígena Wayúu, cuatro (4) estudiantes de procedencia Rural y un (1) estudiante de procedencia Citadina. Con referencia a los estudiantes de procedencia indígena Wayúu, dentro los dibujos y afirmaciones que incluyen elementos propios del entorno natural es posible destacar formas como el sol, el cielo, las nubes, las montañas, diversas frutas como la manzana, el plátano guineo, el maracuyá, el banano, así como las palmas de coco. También sobresalen el mar, la lluvia, innumerables tipos de peces como la Mojarra, el Pargo, el Pechito, la Cacuua, la Boca Colora, el Jurel y el Dormilón. Asimismo, figuran animales como tortugas, delfines y ballenas, entre otros. Estos elementos además de desplegar y precisar una descripción del medio en el cual viven los niños y niñas Wayúu, se ven asociados con la alimentación pues se consideran necesarios para la existencia del sustento alimenticio o se configuran en sí mismos como alimentos integrados a sus dietas.

En el caso de los estudiantes de procedencia Rural, los dibujos y afirmaciones que vinculan descriptores del entorno natural, exhiben elementos como islas, palmas y frutas como el coco, la mandarina y la manzana. Asimismo, aparecen animales como peces entre los que se destacan el Pargo, la Panchita, la Lisa, y el pez Boca Colora, además de cangrejos y medusas. La lluvia también se hace presente junto con el mar y el sol. Finalmente en algunos de los dibujos, los árboles con y sin frutos juegan un papel central y marcan la diferencia con los dibujos de los estudiantes de procedencia indígena Wayúu, pues ninguno de ellos vinculó los árboles en sus dibujos sobre alimentación.

Ahora bien, en la representación de la estudiante de procedencia Citadina es posible apreciar frutas como la manzana, el coco y la mandarina, las cuales son elementos del entorno natural que la rodean y se configuran en sí mismas como alimentos. No obstante, las frutas dibujadas no presentan correspondencia alguna con un árbol, lo cual permite inferir que su concepto de alimentación no entabla relaciones directas entre los alimentos y la naturaleza, debido por ejemplo a que las frutas lleguen a su mesa sin que ella se percate de procesos como el cultivo, la 
Memorias del VII Encuentro Nacional de Experiencias en la Enseñanza de la Biología y la Educación Ambiental y II Congreso Nacional de Investigación en la

\section{Enseñanza de La Biología}

cosecha y la recolección, en los cuales es posible apreciar el ciclo biológico seguido por las platas para arrojar frutos comestibles.

\section{Conclusiones}

El reconocimiento de las representaciones en torno al concepto alimentación es un ejercicio inicial para emprender el replanteamiento tanto de los contenidos de la clase de ciencias como de su forma de enseñanza. Asimismo, éste vislumbra procesos de hibridación cultural frente a los cuales el papel de la escuela resulta decisivo ya sea para adelantar asimilación cultural o empoderamiento y fortalecimiento de la identidad de las comunidades minoritarias que asisten a la escuela y a la clase de ciencias. En este sentido, no es posible iniciar procesos de empoderamiento desde la escuela sin hacer presentes en las clases de ciencias las formas de ver el mundo de grupos culturales minoritarios, por lo cual se hace necesario y urgente su reconocimiento.

Por otra parte, al asumir la perspectiva expuesta en el trabajo de investigación, la presencia de grupos de estudiantes pertenecientes a diversas culturas en el aula de clases permite la negociación cultural, al reconocer la diversidad cultural, hecho que replantea las dinámicas para la enseñanza de las ciencias al enriquecer el trabajo en el aula, pues ahora no se contempla la perspectiva occidental como la única forma válida de ver el mundo. Finalmente, es valioso anotar que la utilización de estos elementos en la construcción de herramientas pedagógicas y didácticas para promover prácticas de buena alimentación en los niños desde la clase de ciencias, teniendo en cuenta un alto sentido cultural, de identidad y de responsabilidad con el otro, dependerá en gran medida de la postura asumida por el maestro.

\section{Bibliografía}

Eco, U. (1953). La estructura Ausente, introducción a la semiótica. Tercera Edición. Lumen S, A. Barcelona, España.

Eco, U. (1986). Segno. Labor, segunda edición. Cartoné.

Geertz, C. (1987). La interpretación de las culturas. México: Editorial Gedisa.

Hall, S. (1997). Representation: Cultural Representations and Signifying Practices. London, Sage Publications, 13-74.

Hewitt, E. (1905). Ethnic Factors in Education. American Anthropologist 6:1-6. 
Bio-grafía Escritos sobre la Biología y su Enseñanza.

Edición Extra-Ordinaria. ISSN 2027-1034 P. p 547-555

Memorias del VII Encuentro Nacional de Experiencias en la Enseñanza de la

Biología y la Educación Ambiental y II Congreso Nacional de Investigación en la Enseñanza de la Biología

Ogbu, J.U. (1982). Cultural Discontinuities and Schooling. In: Anthropology \& Education Quarterly, 13, (4), 290-307.

Parques Nacionales Naturales de Colombia. (2007). Plan De Manejo Santuario De Flora Y Fauna Los Flamencos.

Philips, S. U. (1976). Commentary: Access to Power and Maintenance of Ethnic Identity as Goals of Multi-Cultural Education. In Anthropological Perspectives on Multicultural Education, Anthropology and Education Quarterly, Special Issue. Margaret A. Gibson, ed. 7, (4), 30-32.

Ricoeur, P. (2000). Del texto a la acción. Fondo de Cultura Económica. Argentina.

Ricoeur, P. (2006). Teoría de la interpretación. Discurso y excedente de sentido. Siglo XXI. México.

Riggs, E. M. (2005). Field-Based Education and Indigenous Knowledge: Essential Components of Geoscience Education for Native American Communities. Science Education, 89, (2), 296-313. 